\title{
La carencia de cómics en las bibliotecas universitarias: Estudio de caso del Sistema de Bibliotecas de la Universidad de Puerto Rico
}

\author{
Manuel Martínez Nazario \\ Universidad de Puerto Rico, Recinto de Río Piedras - UPR-RP, Puerto Rico
}

CASE REPORTS

\section{Resumen}

Objetivo. La finalidad de este artículo ha sido documentar los hallazgos de una breve investigación sobre la ausencia de cómics de superhéroes en una biblioteca académica en Puerto Rico, así como demostrar el valor académico de este recurso bibliográfico y la posibilidad de su integración.

Método. Para llevar a cabo este estudio cualitativo, se ha consultado la bibliografía sobre este tema y se han entrevistado a nueve bibliotecarios, con objeto de auscultar las razones del fenómeno.

Resultados. La carencia de cómics de superhéroes guarda relación con la falta de justificación curricular y la ausencia de recomendaciones de adquisición.

Conclusiones. Los cómics de superhéroes han demostrado una gran popularidad entre los lectores y han comprobado su potencial en investigaciones académicas. El alcance de esta fuente de información ha rebasado los límites del entretenimiento y ha logado incorporarse en bibliotecas escolares, públicas y académicas.

Palabras claves

Cómic ; Superhéroe ; Recurso bibliográfico ; Desarrollo de colecciones ; Bibliotecas universitarias ; Currículo ; Investigación ; Puerto Rico

The shortage of superhero comic books in academic libraries: Case study of the Library System of the University of Puerto Rico at Río Piedras

\begin{abstract}
Objective. The objective of this article has been to prove the findings about the lack of superhero comic books in an academic library in Puerto Rico, as in to demostrate its academic value of this bibliographical resource, and the possibility of integration.

Method. For this qualitative study, it has been consulted the related literature, and it has been used interviews, with the intention to explore the reasons of this phenomenon.

Results. The shortage of superhero comic books is connected with the lack of curricular justification and the absence of recommendations for acquisition.

Conclusions. The superhero comic books have demostrated a great popularity among the readers and have comfirmed their potential in the academic researches. The scope of this information source has surpassed the boundaries of entertainment and has been incorporated to school libraries, public libraries and academic libraries.
\end{abstract}

\section{Keywords}

Comic book ; Superhero ; Bibliographic resource ; Collection development ; Academic libraries ; Curriculum ; Research ; Puerto Rico

\section{Introducción}

Los cómics de superhéroes, tanto en versión impresa como digital, han cautivado a millones de personas, a través de los años, en diferentes países del mundo. Su gran popularidad ha trascendido los límites geográficos, ideológicos y lingüísticos, a tal punto de que no son extrañas las traducciones a diferentes idiomas, las 
convenciones de admiradores (Comic-Cons), los productos comerciales, las series televisivas y las obras cinematográficas que se han desarrollado sobre esta temática. En el campo bibliotecológico, las investigaciones sobre la relación entre los cómics de superhéroes y las bibliotecas han sido notables y pertinentes. Sin embargo, son pocas las bibliotecas académicas del mundo que cuentan con este material bibliográfico.

El Sistema de Bibliotecas de la Universidad de Puerto Rico, Recinto de Río Piedras, no dispone de cómics de superhéroes, a pesar de contar con el mayor acervo bibliográfico de la Isla y la mayor variedad temática. La carencia de dicho recurso bibliográfico en sus unidades de información es sorprendente, pues el valor académico de los cómics de superhéroes ha sido evidenciado en múltiples obras de crítica e interpretación, particularmente en las diversas tesis publicadas en Estados Unidos, Canadá y Reino Unido (ProQuest, 2014). Esta premisa ha sido la promotora de este breve trabajo investigativo, el cual formó parte de los requisitos del curso Metodología de la Investigación para Profesionales de la Información (CINF 6706), de la Escuela Graduada de Ciencias y Tecnologías de la Información de la Universidad de Puerto Rico, Recinto de Río Piedras. Para ello, se propuso conocer las causas de este fenómeno, documentar los hallazgos, demostrar su valor académico y justificar la inclusión de los cómics de superhéroes en el Sistema de Bibliotecas. Pero antes de pasar al tema principal de este adelanto de investigación, resulta necesario saber qué es un cómic y qué es un superhéroe, aunque sea de manera concisa.

\section{Definiciones}

McCloud (1994) ha definido los cómics como un medio donde se combina de forma deliberada texto e imágenes con la intención de transmitir información. Por su parte, el famoso escritor Stan Lee ha destacado el valor artístico de los cómics al expresar lo siguiente:

If Shakespeare and Michelangelo were alive today, and if they decided to collaborate on a comic, Shakespeare would write the script and Michelangelo would draw it. How could anybody say that this wouldn't be as worthwhile an art form as anything on earth? (McLaughlin, 2007, p. 105).

Por otro lado, Klock (2013) ha manifestado que definir el concepto superhéroe es complejo; pero aun así podría definirse como un personaje fantástico que posee poderes que exceden los términos de la naturaleza humana con el propósito de luchar contra la maldad, defendiendo a los más débiles por medio de acciones heroicas. Por lo tanto, y muy de acuerdo con Hatfield, Heer y Worcester (2013), los cómics son una forma de arte y los superhéroes son el género.

\subsection{Trasfondo del problema}

Con el propósito de auscultar la disponibilidad de cómics de superhéroes en las colecciones del Sistema de Bibliotecas de la Universidad de Puerto Rico, se realizó una búsqueda en el catálogo en línea utilizando las opciones Palabra Clave General (General Keyword), Palabra Clave Materia (Subject Keyword), Búsqueda Alfabética por Título (Title Browse) y Palabra Clave Título (Title Keyword), bajo los siguientes títulos: Batman, Captain America, Fantastic Four, Flash, Green Lantern, Hulk, Iron Man, Spiderman, Superman, Thor, Wonder Woman y $X-M e n$. Estás búsquedas no produjeron ningún resultado.

\subsection{Declaración del problema}

Los hallazgos de esta búsqueda produjeron varias interrogantes. ¿Por qué no hay cómics de superhéroes en las colecciones? ¿Cuáles son las razones? La carencia de cómics de superhéroes en las colecciones del Sistema de Bibliotecas reduce la posibilidad de descubrir nuevos temas de investigación académicas. Este género literario encierra una gran fuente de información que no ha sido explotada al máximo, o por lo menos, considerada por los catedráticos y bibliotecarios del Recinto de Río Piedras. No obstante, en 2013 se presentó, en el Departamento de Historia de la Facultad de Humanidades, la primera tesis de maestría sobre la relación entre los cómics de superhéroes y el discurso militar de Estados Unidos en el siglo XXI (Serrano, 2013). Por lo tanto, ¿se justifica la inclusión de cómics de superhéroes en el Sistema de Bibliotecas? 


\subsection{Justificación del estudio}

La incorporación de cómics de superhéroes a las colecciones del Sistema de Bibliotecas, podría brindar a los estudiantes mayores fuentes de investigación. Por ejemplo, podrían analizarse los discursos políticos, los problemas sociales, las influencias filosóficas, las variantes lingüísticas y los trastornos psicológicos presentados por escritores y dibujantes de este recurso bibliográfico, tales como: Geoff Johns, Bob Kane, Jack Kirby, Jim Lee, Stan Lee, Jeph Loeb, Frank Miller, Alan Moore, Grant Morrison y Jerry Siegel. Para Hoppenstand (2014), los cómics de superhéroes tienen otros valiosos elementos que deben analizarse profundamente, tales como: el concepto de continuidad, el problema racial, el prejuicio de género y la identidad estereotipada. Por otra parte, Klock (2002) ha destacado la relación de este género literario con la mitología estructural, la historia cultural y los estudios culturales.

La Facultad de Humanidades de la Universidad de Puerto Rico en Río Piedras tiene un Programa de Estudios Interdisciplinarios que ofrece a sus estudiantes la oportunidad de traspasar las fronteras de diversas disciplinas para crear su propia concentración de estudios. Los cómics de superhéroes podrían suplir esa necesidad de información con objeto de crear nuevos conocimientos. Los estudiantes de la Facultad de Ciencias Sociales también podrían beneficiarse de este recurso bibliográfico, pues dicha facultad dispone de áreas de estudios, tales como: antropología, sociología, psicología y ciencia política. Otras disciplinas que podrían utilizar dicho recurso bibliográfico serían: artes plásticas, comunicación, drama, filosofía, historia del arte, idiomas, lingüística y literatura comparada. Varias universidades en Estados Unidos han establecido un precedente en cuanto al uso de cómics como recurso curricular en cursos de psicología, literatura comparada, estudios interdisciplinarios, sociología y comunicación (O’English, 2009).

\subsection{Delimitaciones del estudio}

Este trabajo aborda solamente el cómic de superhéroe, ya que existen otras categorías tales como: aventura, crítica social, deporte, detectivesca, erótica, pícara, política, religión y romance. Además, se ha decidido delimitar su alcance al Sistema de Bibliotecas de la Universidad de Puerto Rico, Recinto de Río Piedras, porque el Sistema de Bibliotecas posee el acervo bibliográfico más grande y variado de todo Puerto Rico, y el Recinto de Río Piedras dispone de la mayor cantidad de programas académicos en sus ofertas de bachillerato, maestría y doctorado.

\section{Revisión de literatura}

O’English (2009) ha declarado que los cómics tienen la capacidad de traspasar las fronteras disciplinarias y rebasar los límites tradicionales de los procedimientos técnicos y del servicio público. Esta bibliotecaria de la Universidad Estatal de Washington ha manifestado que los cómics se acomodan a las enseñanzas académicas de cuatro formas: como objeto de estudio, como apoyo al currículo, como desarrollo personal y profesional y como lectura recreativa. Sus argumentos se han amparado en dos estudios de casos realizados en la Universidad Estatal de Washington y la Universidad de Memphis, y en la literatura especializada en el tema de cómics.

Laycock (2005), maestra-bibliotecaria en Barker College de Sidney, Australia, ha destacado la gran importancia de desarrollar una colección de cómics de manera racional. Para ello, ha esbozado detalladamente ocho criterios: distribución de presupuesto, selección, catalogación, localización, exposición, promoción, mantenimiento y apoyo a la colección. Además, la autora ha argumentado sobre la naturaleza, la importancia y el alcance de estos recursos bibliográficos en las bibliotecas académicas.

Por su parte, Lavin (1998) ha reconocido que la mayor parte de las colecciones en Estados Unidos no consideraban los cómics como recursos bibliográficos complejos que pueden aportar al desarrollo de aptitudes entre los estudiantes. También, dicho autor ha reconocido que, además de un simple entretenimiento, los cómics pueden ser objeto de estudios académicos en varias disciplinas. Lavin, quien es bibliotecario en la Universidad del Estado de Nueva York en Buffalo, ha sugerido 10 criterios de selección de cómics, los cuales servirían de guía a los bibliotecarios a cargo de esta colección: popularidad, idoneidad, edad del lector, género, calidad de trama, calidad artística, estilo artístico, reputación de los escritores, premios o reconocimientos que la obra ha recibido, y formato.

Asimismo, Scott (1998) ha confirmado la viabilidad de desarrollar una colección de cómics en una biblioteca académica, específicamente en la Universidad Estatal de Michigan, la cual sobrepasó los 100,000 ejemplares en 1998. Según el autor, la automatización de la catalogación, los esfuerzos cooperativos entre bibliotecas y el 
interés de los bibliotecarios han contribuido a la incorporación de los cómics a algunas bibliotecas. A diferencia de Laycock y Lavin, Scott, quien se ha desempeñado como bibliotecario catalogador y colaborador en el desarrollo de la colección de cómics en la Universidad Estatal de Michigan, ha presentado casos concretos de investigaciones interdisciplinarias que han utilizado los cómics como fuente de información.

Cubbage (2010), bibliotecaria coordinadora de las Humanidades y especialista en la materias de inglés, literatura comparada y actuación, de la Biblioteca de la Universidad de Northwestern en Illinois, ha demostrado cómo los estudios interdisciplinarios sobre cultura popular han ido aumentando a través de los años, siendo el cómic un excelente vehículo de transmisión. Según la autora, desde 1989 hasta 2010 se han publicado en Estados Unidos 27 tesis enfocadas en cómics. A diferencia de los autores antes mencionados, Cubbage ha tocado el tema de la integración curricular universitaria y para ello ha recomendado el análisis de los intereses de investigación de los estudiantes por medio de encuestas o entrevistas. Además, ha advertido a los bibliotecarios sobre la necesidad de conocer plenamente cuáles son las metas y los objetivos institucionales, pues el éxito de la colección dependerá de cuán integrados se encuentren con el programa educativo.

Por último, la Biblioteca Ignasi Iglésias-Can Fabra (2008), del Distrito de Sant Andreu, en Cataluña, ha presentado, en su ponencia titulada La entrada del cómic en las bibliotecas, algunos datos estadísticos que demuestran el crecimiento de la colección de cómics y de su uso en la biblioteca entre 2005 y 2007 . Los resultados estadísticos, en los que se distingue el cómic infantil del cómic para adultos, han demostrado la acogida de dicho recurso bibliográfico en una biblioteca pública española. También, en dicha ponencia se ha destacado las actividades realizadas en dicho peridodo, tales como: exposiciones, club de lectura, talleres, presentaciones, conferencias, charlas y cursos de colaboración.

\section{Metodología}

Para llevar a cabo esta investigación se ha utilizado un enfoque cualitativo, ya que se han recopilado datos descriptivos que han sido analizados mediante medios no matemáticos. La investigación es, además, inductiva, ya que ha partido de unas interrogantes previamente establecidas y el tema se ha desarrollado a partir de la recopilación de datos. El alcance de este estudio ha sido explicativo, pues ha tenido como objetivo identificar y explicar las causas de un fenómeno (Hernández, Fernández y Baptista, 2010); o sea, describir, comprender e interpretar la realidad sobre la carencia de cómics de superhéroes en el Sistema de Bibliotecas, de la Universidad de Puerto Rico, Recinto de Río Piedras. Como parte del estudio, se llevaron a cabo entrevistas estructuradas, en octubre de 2014, a nueve bibliotecarios del Sistema de Bibliotecas relacionados con la adquisición, el desarrollo de colecciones, la catalogación y el servicio de referencia especializado en arte.

\section{Recopilación de datos}

Para la realización de las entrevistas, se formularon una serie de preguntas sobre asuntos relacionados con el desarrollo de colecciones, ya sea mediante compra, intercambio o donaciones, y la percepción y la apreciación de los bibliotecarios en torno a los cómics de superhéroes. Las preguntas incluidas en la entrevista fueron las siguientes: ¿Por qué cree usted que el Sistema de Bibliotecas no tiene cómics de superhéroes?, ¿Contempló en algún momento el Sistema de Bibliotecas la adquisición de cómics de superhéroes?, ¿Recomendó alguien la adquisición de cómics de superhéroes?, ¿Recibió en algún momento el Sistema de Bibliotecas, por medio de donación o intercambio, cómics de superhéroes?, ¿Por qué cree usted que sea necesario o innecesario la adquisición de cómics de superhéroes?, ¿En cuál colección deben estar localizados los cómics de superhéroes?, ¿Sabe usted de alguna biblioteca en Puerto Rico que tenga cómics de superhéroes?, ¿Qué efecto podría tener la carencia de cómics de superhéroes en las colecciones del Sistema de Bibliotecas?, ¿Ha leído usted en algún momento cómics de superhéroes?, En caso de responder negativamente, ¿por qué no ha leído usted cómics de superhéroes?, ¿Cuál o cuáles cómics de superhéroes ha leído usted?, ¿Qué te gusta de los cómics de superhéroes?, y ¿Qué no te gusta de los cómics de superhéroes?

\section{Resultados}

Según seis bibliotecarios entrevistados, el Sistema de Bibliotecas de la Universidad de Puerto Rico, Recinto de Río Piedras, no dispone de cómics de superhéroes en su acervo porque no son parte del currículo académico universitario. Por otro lado, dos bibliotecarios declararon que la ausencia de dicho recurso bibliográfico en las colecciones se debió a la falta de recomendaciones, mientras que un bibliotecario señaló que el Sistema de Bibliotecas no adquirió cómics de superhéroes porque se les consideró poco serio. Además, el Sistema de 
Bibliotecas recibió por medio de donación, en la década de los 80, una indeterminada cantidad de cómics y estos, a su vez, fueron donados a una biblioteca pública.

\begin{tabular}{|l|c|c|}
\hline \multicolumn{1}{|c|}{ Respuestas } & Entrevistados & Porciento \\
\hline No eran parte del currículo académico & 6 & $67 \%$ \\
\hline No fueron recomendados para adquisición & 2 & $22 \%$ \\
\hline No se consideró un recurso bibliográfico serio & 1 & $11 \%$ \\
\hline
\end{tabular}

Tabla 1 - Razones por las cuales el Sistema de Bibliotecas no tiene cómics de superhéroes

Todos los bibliotecarios entrevistados opinaron que los cómics de superhéroes son necesarios porque son una valiosa fuente de información, podrían enriquecer algunos programas académicos, contribuirían al desarrollo de algunas destrezas (abstracción, asociación de ideas, inferencia, lectura y secuencia) y servirían de entretenimiento.

\begin{tabular}{|l|c|c|}
\hline \multicolumn{1}{|c|}{ Respuestas } & Entrevistados & Porciento \\
\hline Apoyo a programas académicos & 4 & $45 \%$ \\
\hline Desarrollo de destrezas & 1 & $11 \%$ \\
\hline Entretenimiento & 1 & $11 \%$ \\
\hline Valiosa fuente de información & 3 & $33 \%$ \\
\hline
\end{tabular}

Tabla 2 - Valor de los cómics de superhéroes

Por otro lado, siete bibliotecarios indicaron que la carencia de cómics de superhéroes limita la diversidad temática de las colecciones existentes, reduciendo las fuentes de información necesarias para la investigación académica, mientras que dos de los entrevistados no pudieron identificar los posibles efectos de la falta de cómics de superhéroes.

\begin{tabular}{|l|c|c|}
\hline \multicolumn{1}{|c|}{ Respuestas } & Entrevistados & Porciento \\
\hline Limita la diversidad temática & 7 & $78 \%$ \\
\hline No se puede precisar & 2 & $22 \%$ \\
\hline
\end{tabular}

\section{Tabla 3 - Efectos de la carencia de cómics de superhéroes}

En cuanto al lugar donde deben ubicarse los cómics de superhéroes, cinco bibliotecarios consideraron la Colección de la Artes, mientras que los cuatro bibliotecarios restantes consideraron la Colección de Circulación, la Biblioteca de Comunicación Pública, la Biblioteca de Educación y la Biblioteca de Ciencias Bibliotecarias e Informática. Estas dos últimas bibliotecas fueron consideradas por los entrevistados porque ya cuentan con una colección de libros de índole juvenil. Por otra parte, los bibliotecarios entrevistados indicaron desconocer si existe una colección de cómics de superhéroes en alguna biblioteca pública o privada en Puerto Rico.

\begin{tabular}{|l|c|c|}
\hline \multicolumn{1}{|c|}{ Respuestas } & Entrevistados & Porciento \\
\hline Biblioteca de Ciencias Bibliotecarias e Informática & 1 & $11 \%$ \\
\hline Biblioteca de Comunicación Pública & 1 & $11 \%$ \\
\hline Biblioteca de Educación & 1 & $11 \%$ \\
\hline Colección de Circulación & 1 & $11 \%$ \\
\hline Colección de las Artes & 5 & $56 \%$ \\
\hline
\end{tabular}


Todos los bibliotecarios entrevistados leyeron en algún momento cómics de superhéroes y consideraron la lectura un mero recreo; siendo Batman, Spiderman y Superman los más populares entre ellos. Los elementos que más gustaron de la lectura de cómics de superhéroes fueron: la narración, la acción, la intriga, los colores llamativos, los dibujos, las historias, el carácter fantástico, la lucha entre el bien y el mal, y las ideas presentadas en forma tácita. Por otro lado, los elementos que menos gustaron fueron: la violencia, lo grotesco de las figuras humanas, la carga ideológica y la interrupción en el relato con objeto de continuarlo en el próximo ejemplar.

\begin{tabular}{|c|c|c|}
\hline Respuestas & Entrevistados & Porciento \\
\hline El pensamiento tácito & 1 & $11 \%$ \\
\hline La acción & 1 & $11 \%$ \\
\hline La intriga & 1 & $11 \%$ \\
\hline La lucha entre el bien y el mal & 1 & $11 \%$ \\
\hline La narración & 1 & $11 \%$ \\
\hline La resolución de problemas & 1 & $11 \%$ \\
\hline Las historias & 1 & $11 \%$ \\
\hline Los elementos fantásticos & 1 & $11 \%$ \\
\hline Los elementos gráficos & 1 & $11 \%$ \\
\hline
\end{tabular}

Tabla 5 - Los elementos que más gustaron de los cómics de superhéroes

\begin{tabular}{|c|c|c|}
\hline Respuestas & Entrevistados & Porciento \\
\hline El elevado precio de los cómics & 1 & $11 \%$ \\
\hline La carga ideológica & 1 & $11 \%$ \\
\hline La interrupción del relato & 1 & $11 \%$ \\
\hline La violencia & 2 & $22 \%$ \\
\hline Las explosiones & 1 & $11 \%$ \\
\hline Lo grotesco en las figuras humanas & 1 & $11 \%$ \\
\hline Nada & 2 & $22 \%$ \\
\hline
\end{tabular}

Tabla 6 - Los elementos que menos gustaron de los cómics de superhéroes

Al final de la entrevista, y a modo de comentario, siete de los bibliotecarios recalcaron que los cómics de superhéroes tienen un valor académico, puesto que son una fuente valiosa de información y que podrían ayudar a los estudiantes a seleccionar nuevos temas de investigación.

\section{Conclusiones}

La carencia de cómics de superhéroes en el Sistema de Bibliotecas de la Universidad de Puerto Rico, Recinto de Río Piedras, es constatable y se debe principalmente, según los bibliotecarios entrevistados, a la falta de justificación curricular y a la ausencia de recomendaciones de adquisición. No obstante, otros recursos bibliográficos similares han sido adquiridos en el Sistema de Bibliotecas, tales como: Toda Mafalda, de Quino (1997), The complete Peanuts, de Schulz (2004) y Turey el Taíno, de Álvarez Rivón (1995). En la Política de Desarrollo de Colecciones, del Sistema de Bibliotecas (1997), no existe ninguna disposición que excluya el recurso bibliográfico en discusión. En caso de una estrechez presupuestaria institucional, la adquisición de cómics de superhéroes por medio de donaciones o intercambio entre bibliotecas se perfilarían como posibles alternativas. Para ello, sería necesario trazar un plan estratégico que permitar identificar los contactos y establecer los convenios de intercambio o donaciones.

Para configurar una colección de cómics de superhéroes coherente y atractiva sería necesario consultar la literatura bibliotecológica en torno a los cómics. Goldsmith (2009), Highsmith (1992), Scott (1990), Weiner (2010) 
y Young (2014) han delineado los parámetros de desarrollo de colecciones de cómics para bibliotecas, tomando en cuenta la evaluación, adquisición, catalogación, localización, preservación y promoción. Estos parámetros podrían ser utilizados como marco de referencia al momento de crear una colección con las características antes mencionadas, la cual satisfaría las posibles necesidades de sus usuarios y enriquecería el acervo bibliográfico del Sistema de Bibliotecas. En Estados Unidos, algunas bibliotecas académicas han logrado establecer colecciones de cómics, las cuales podrían ser utilizadas como modelos por el Sistema de Bibliotecas. Entre las bibliotecas que disponen de dichos recursos en su acervo se destacan la Universidad Estatal de Michigan, la Universidad de Indiana, la Universidad de California en Los Ángeles y Riverside, y la Universidad de Pittsburgh (Scott, 1990).

A diferencia de los casos presentados por DeCandido (1991), Pyles (2012) y Wilkins (2004), en los que se ha destacado la renuencia de los bibliotecarios a incluir a los cómics de superhéroes en las bibliotecas académicas, los bibliotecarios del Sistema de Bibliotecas reconocieron el gran valor que encierra dicha publicación, el potencial en el desarrollo de destrezas, la conveniencia, tanto para las colecciones bibliográficas como para las investigaciones académicas, y la posibilidad de entretenimiento para sus lectores. Russell (2013) ha destacado dicha dualidad (valor académico-entretenimiento) y ha declarado lo siguiente: “...comic books deliver to readers information that they will not only enjoy, but also give them a staging ground from which they can take away something more." (p. 122). Esta buena percepción, por parte de los bibliotecarios entrevistados, facilitaría la incorporación de los cómics de superhéroes a las colecciones del Sistema de Bibliotecas, a diferencia de lo presentado por Aros, Díaz, Naranjo, Riveros y Toledo (2013), y Fernández y Bravo (2009), quienes han mencionado algunas de las barreras, mitos y prejuicios sobre los cómics, que se palpan entre los profesionales de la información, y que inciden en el desarrollo de una colección de cómics.

La tesis de maestría de Serrano Denis (2013), la cual fue presentada en el Departamento de Historia, de la Facultad de Humanidades, ha demostrado la necesidad y la importancia de los cómics de superhéroes y establece un precedente académico en Puerto Rico. El Sistema de Bibliotecas cuenta con los recursos necesarios para la adquisición, catalogación, preservación, promoción y localización de dicho material impreso. Una vez adquirido los cómics de superhéroes, la promoción bibliotecaria, a través de la página electrónica del Sistema de Bibliotecas, sería vital y conveniente, pues ayudaría a estimular la creación de cursos o la producción de investigaciones.

Los cómics de superhéroes han demostrado una gran popularidad como género literario y un considerable potencial en investigaciones académicas. El alcance de este recurso bibliográfico ha rebasado los límites del entretenimiento y ha logrado incorporarse, de forma progresiva, en bibliotecas escolares, públicas y universitarias en Australia, Canadá, España y Estados Unidos. Este breve trabajo investigativo ha demostrado que los cómics de superhéroes pueden tener un espacio en el Sistema de Bibliotecas, pues dicho recurso bibliográfico puede cumplir una triple finalidad: ampliar las posibilidades de estudio, enriquecer el acervo bibliográfico y ofrecer a sus usuarios una alternativa de esparcimiento.

\section{Agradecimientos}

El autor da las gracias al Dr. José A. Sánchez Lugo, de la Escuela Graduada de Ciencias y Tecnologías de la Información, de la Universidad de Puerto Rico, Recinto de Río Piedras, por su interés y apoyo en esta investigación. Asimismo, el escritor agradece a la Dra. Marilyn Montalvo Montalvo, del Sistema de Bibliotecas de la Universidad de Puerto Rico, Recinto de Río Piedras, por sus diligentes correciones y por sus acertadas sugerencias. 


\section{Referencias}

Álvarez Rivón, R. (1995). Turey el Taíno. San Juan: Instituto de Cultura Puertorriqueña.

Aros Ahumada, M., Díaz Jara, C., Naranjo Reyes, B., Riveros Basoalto, G., y Toledo Figueroa, R. (2013). El cómic en las bibliotecas públicas: Propuesta de difusión web para bibliotecas y lectores. Serie Bibliotecológica y Gestión de Información. 84, $1-44$.

Biblioteca Ignasi Iglésias-Can Fabra. (2008). La entrada del cómic en las bibliotecas: Tratando todos los géneros, Ilegando a todos los públicos... ampliando servicios. En IV Congreso Nacional de Bibliotecas Públicas. A Coruña 24, 25, 26 de septiembre de 2008. Madrid: Ministerio de Cultura, Dirección General del Libro, Archivos y Bibliotecas, Subdirección General de Coordinación Bibliotecaria.

Cubbage, C. (2010). Selection and popular culture in large academic libraries: Taking the temperature of your research community. En R. G. Weiner, Graphic novels and comics in libraries and archives: Essays on readers, research, history and cataloging (72-80). Jefferson: McFarland \& Company.

DeCandido, K.R.A. (1991). Get the picture? A serious look at comics in libraries. Library Journal, 116, 46-50.

Dewey, M., Mitchell, J.S., Beal, J., Green, R., Martin, G., y Panzer, M. (2011). Dewey decimal classification and relative index. Tomo 3. Dublin, Ohio: Online Computer Library Center.

Fernández Fernández, S., y Bravo, P.A. (2009). Cómic, lectura y bibliotecas. La Biblioteca Central Tecla Sala de L'Hospitalet. Boletín de la Asociación Andaluza de Bibliotecarios, 94-95, 105-114.

Goldsmith, F. (2009). A place in the library. En M. Cornog y T. Perper, Graphic novels beyond the basics: Insights and issues for libraries (pp. 141-161). Santa Barbara: ABC CLIO.

Hatfield, C, Heer, J., y Worcester, K. (2013). The superhero reader. Jackson: University Press Mississppi.

Hernández Sampieri, R., Fernández Collado, C., y Baptista Lucio, M.P. (2010). Metodología de la investigación. México: McGraw Hill.

Highsmith, D. (1992). Developing a "Focused" comic book collection in an academic library. En A.W. Ellis, Popular culture and acquisitions (59-68). Nueva York: Haworth Press.

Hoppenstand, G. (2014). The graphic novel. Ipswich, Massachusetts: Salem Press.

Klock, G. (2002). How to read superhero comics and why. Nueva York, Londres: Continuum.

Klock, G. (2013). What is a superhero? No one knows-That's what makes 'em great. En R.S. Rosenberg y P.M. Coogan, P.M., What is a superhero? (pp. 71-75). Nueva York: Oxford University Press.

Lavin, M.R. (1998). Comic books and graphic novels for libraries: What to buy. Serials Review, 24(2), 31-45. doi:

10.1080/00987913.1998.10764444

Laycock, D. (2005). Developing a graphic novel collection. Synergy, 3(2), 50-54.

McCloud, S. (1994). Understanding comics: the invisible art. Nueva York: Harper Perennial.

McLaughlin, J. (2007). Stan Lee: Conversations. Jackson: University Press of Mississippi.

O’English, L. (2009). Comics and graphic novels in the academic library collection. En M. Cornog y T. Perper, Graphic novels beyond the basics: Insights and issues for libraries (pp. 163-189). Santa Barbara: ABC CLIO.

ProQuest. (2014). Dissertations Express. Recuperado de http://dissexpress.umi.com/

Pyles, C. (2012). It's no joke: Comics and collection development. Public Libraries, 51(6), 32-35.

Quino. (1997). Toda Mafalda. Buenos Aires: Ediciones de la Flor.

Russell, C. (2013). Heroic moments: A study of comic book superheroes in real-world society. Explorations, 8, 121-131.

Schulz, C.M. (2004). The complete Peanuts. Seattle: Fantagraphics Books.

Scott, R.W. (1990). Comics librarianship: A handbook. Jefferson: McFarland \& Company.

Scott, R.W. (1998). A practicing comic-book librarian surveys his collection and his craft. Serials Review, 24(1), 49-56. doi: 10.1080/00987913.1998.10764429 
Serrano Denis, R.A. (2013). Avenging the past: Cold war revival and the saving of military triumphalism by comic book superheroes in post 9/11 America. Thesis (M.A.): Universidad de Puerto Rico.

Sistema de Bibliotecas. Recinto de Río Piedras. Universidad de Puerto Rico. (1997). Política de Desarrollo de Colecciones. Recuperado el 15 de octubre de 2014 de http://biblioteca.uprrp.edu/Politicas/Politica-DesCol.htm.

Weiner, R.G. (2010). Graphic novels and comics in libraries and archives: Essays on readers, research, history and cataloging. Jefferson: McFarland \& Company.

Wilkins, D. (2004). Graphic novels and comics in libraries. Indiana Libraries, 23(2), 17-18.

Young, B. (2014). Wham! Pow! Discussion of a graphic novel and comic book core collection in an academic library. Mississippi Libraries, 77(1), 9-11.

\section{Datos del autor}

Manuel Martínez Nazario

Estudiante de la Maestría de la Escuela Graduada de Ciencias y Tecnología de la Información. Universidad de Puerto Rico, Recinto de Río Piedras - UPR-RP. Se desempeña como bibliotecario a cargo de la Oficina de Préstamos Interbibliotecarios de la Colección de Referencia del Sistema de Bibliotecas UPR.

manuel.martinez8@upr.edu

$\begin{array}{ll}\text { Recibido - Received } & : 2014-12-30 \\ \text { Aceptado-Accepted } & : 2015-03-25\end{array}$

